\title{
REMARKS ON BALANCED INCOMPLETE BLOCK DESIGNS
}

\author{
E. T. PARKER
}

A balanced incomplete block design (BIBD) is a class of $b$ subsets, or blocks, from a set of $v$ elements with $k$ elements in each block; each element is in $r$ blocks and each pair of distinct elements is in $\lambda$ blocks. We shall establish the following

Theorem. Let $D$ be a $B I B D$ with parameters $(v, b, k, r, \lambda)=(2 x+2$, $4 x+2, x+1,2 x+1, x)$, where $x$ is an even positive integer. Then (i) any two blocks of $D$ have at least one common element; (ii) no two blocks of $D$ are the same subset.

Proof. Assume the hypothesis and the falsity of either conclusion. Construct matrix $A$ of $2 x+2$ rows and $4 x+4$ columns, with entries +1 and -1 . The first column contains exclusively +1 , and the second column -1 . Set up one-to-one correspondences between rows of $A$ and elements of $D$; between columns other than the first two of $A$ and blocks of $D$. Enter +1 if the element is contained in the block and -1 otherwise. Then each row of $A$ contains exactly $1+0$ $+(2 x+1)=2 x+2$ entries +1 , and hence $2 x+2$ entries -1 . Further, each pair of distinct rows contains two +1 's in exactly $1+0+x=x+1$ like columns. It follows that each pair of distinct rows of $A$ has in like columns the ordered pairs $(1,1),(1,-1),(-1,1)$, and $(-1,-1)$ each $x+1$ times. Select $x+1$ rows corresponding to the elements of a block which is either repeated in $D$ or disjoint from another block of $D$. Let $A_{0}$ be the $x+1$ by $4 x+4$ submatrix of $A$ composed of these rows. Then $A_{0} A_{0}^{T}=(4 x+4) I$, with the identity matrix of dimension $x+1 . A_{0}$ has four columns each with all entries equal; these are the first two and those corresponding to the pair of special blocks of $D$. All pairs of unequal \pm 1 entries in like columns of $A_{0}$ therefore occur in the other $4 x$ columns. Each pair of distinct rows of $A_{0}$ contains $2 x+2$ unlike entries in like columns; thus the total number of pairs of unequal entries within columns is $(x+1) x \cdot(2 x+2) / 2=(x+1)^{2} x$. Among the $4 x$ columns the average number of unlike pairs is accordingly $(x+1)^{2} x / 4 x$ $=(x+1)^{2} / 4$. A partition of $x+1$ elements into two classes, with as many as $(x+1)^{2} / 4$ pairs of elements not in the same class, is possible only if $x+1$ is even.

There exist numerous examples of BIBD with parameters as in the theorem and $x$ an odd positive integer. (It is likely in fact that a design exists for each choice of $x$. This would be a corollary of Paley's

Received by the editors June 21, 1962. 
very plausible conjecture that a so-called Hadamard matrix exists for order each multiple of four. Such a matrix has elements \pm 1 and all inner products zero between distinct rows (see [1] and bibliography thereof). The converse implication might be false.) For odd $x$ conclusion (i) of the theorem need not be true; an example is outlined for $x=3$. Form the symmetric and cyclic BIBD with parameters $(v, b, k, r, \lambda)=(15,15,7,7,3)$ determined by the difference set $0,1,2,4,5,8,10(\bmod 15)$. Delete one block and all its elements where they occur in the other blocks, leaving a BIBD with parameters $(8,14,4,7,3)$. It is easily verified that the latter BIBD has a pair (in fact seven pairs) of blocks without common element.

Whether conclusion (ii) holds for odd $x$ appears less easy to decide; the above proof is not valid, but the author has constructed no counterexample. It is seldom if ever stated explicitly that all blocks of a BIBD must be distinct. Examples of BIBD are constructed easily with pairs of like blocks by choosing a quintuple of parameters for which a solution exists, then multiplying $b, r$, and $\lambda$ by an integer greater than one; it is rather understood that $b, r$, and $\lambda$ should not have a common prime divisor, for this bad property means in effect that a designed experiment would be doubled, tripled, etc., in size. Even for $b, r, \lambda$ lacking a common divisor, there exist BIBDs with pairs of like blocks. An example is presented with parameters $(10,30,3,9,2)$. The ten elements are designated by $X, Y, Z ; 0,1,2$, $3,4,5,6$. The thirty triples are $X Y Z$ twice; and four classes of seven blocks each obtained by adding $(\bmod 7)$ all constants to the digits in $X 01, Y 02, Z 04$, and 124 , leaving $X, Y, Z$ fixed by the addition. Thus the second half of the theorem is not vacuous in content.

\section{REFERENCE}

1. Leonard Baumert, S. W. Golomb, and Marshall Hall, Jr., Discovery of an Hadamard matrix of order 92, Bull. Amer. Math. Soc. 68 (1962), 237-238.

UNIVAC Division of Sperry Rand Corporation, St. Paul, Minnesota 